\title{
The Medanta AOLO template for locally advanced cancer cervix brachytherapy: design and clinical implementation
}

\author{
Susovan Banerjee, MD, Venkatesan Kaliyaperumal, MSC, Tejinder Kataria, MD, DNB, Dayanidhi Kamaraj, MSC \\ Division of Radiation Oncology, Medanta - The Medicity, Gurgaon, Haryana, India
}

\begin{abstract}
Purpose: In advanced cervical cancers, improved techniques using both intracavitary and interstitial brachytherapy (IC + IS) should be used to adequately cover the residual tumor volume, resulting in better clinical results. Confronted with some limitation in implementing available applicators, we devised our own indigenous template that would also serve as an applicator.

Material and methods: First, a graphical design was drawn, keeping in mind the extent of volumes that need to be targeted in various brachytherapy scenarios. It was validated in a computer system and a physical template was manufactured. The template underwent modified versions through iterative dosimetric and planning exercises. The final model was successfully implemented in a clinical scenario.

Results: Cylindrical templates of diameter 3, 4, and $5 \mathrm{~cm}$ were produced with central aperture for central tandem, combining surrounding holes spaced at pre-planned points. The instrument can be used for IC + IS brachytherapy in cervical cancers. This easy to use applicator/template can direct needles to treat a distance of $4.5 \mathrm{~cm}$ laterally at the level of point A. In two initial applications 55 and $54 \mathrm{cc}$ of high-risk clinical target volumes (HRCTV) were treated, and $90 \%$ of the HRCTV was covered by $96 \%$ and $97 \%$ of prescribed doses, respectively. Additionally, the needles guided by the template helped in sculpting the dose from bladder and rectum.

Conclusions: We have developed an easy to use IC + IS type of template/applicator, which was successfully implemented in our clinical practice.

J Contemp Brachytherapy 2020; 12, 1: 44-47 DOI: https://doi.org/10.5114/jcb.2020.92528
\end{abstract}

Key words: cervical cancer, indigenous template, Medanta AOLO.

\section{Purpose}

TheGynecological(GYN)GEC-ESTROWorkingGroup guidelines and the ICRU report 89 has standardized the approach of image-guided adaptive brachytherapy (IGABT) in locally advanced cervical cancer (LACC) $[1,2]$. The core of IGABT is an assessment of tumor response during radiotherapy and an adaptive tailoring of brachytherapy boost according to the individual tumor response. The disease and normal structures can be contoured, and a curable dose can be delivered while avoiding the critical structures. Depending on disease extent, a simple intracavitary application (IC), along with/without interstitial needles (IS), can be performed. It is more important to use IGABT in cases where interstitial needles are used to treat the lateral parametrium in LACC. IGABT is now successfully implemented in clinical practice, with rewarding results in terms of local control of disease and disease-free survival [2].
However, a wide spread adoption of IGABT is difficult due to high cost and fragility of applicators [3]. Quality brachytherapy application requires an investment in equipment as well as skills and expertise, which can pose a challenge especially in the developing countries $[4,5]$. We faced similar difficulties in implanting IGABT at our institute, so we developed our own template that can also serve as an applicator.

\section{Material and methods}

The template/applicator was constructed with some advantages like a single piece of applicator, which can accommodate the central tandem along with the straight needles that can be used directly. It should allow IC + IS type of loading and the $200 \%$ of the dose to be confined within the applicator. It was intended that the applicator should be easy to use, hence having minimal learning curve. 
The Medanta anterior oblique lateral oblique (AOLO) template uses currently existing and commercially available Nucletron-Elekta intrauterine tubes of 15, 30, and 45 degree as central tandem through the central hole, and 6 F catheters of the Kalyani Radiotherapy Specialty India (P) Ltd. for other holes in pre-planned fixed coordinates of the template.

Initial conceptualization of the applicator came from a study on plotting the width and thickness of high-risk clinical target volume (HRCTV) at multiple levels starting from cervical os [6]. With the width and thickness of HRCTV available at different levels, the number and divergence of needles were drawn on a graph paper in a 2 dimensional concept. At the same time, models were created in the treatment planning systems, and trials were done on the length, angle, and numbers of needles that could be accommodated in a cylinder of $4 \mathrm{~cm}$ diameter. Possible ranges of HRCTV were drawn and the coverage was validated. After many iterations, the actual template was created. Further experimentations and modifications were done to derive the final version that was available for clinical use [7]. There are entry and exit points of 3 anterior oblique catheters $(\mathrm{AO})$ in direction of intrauterine part of the central tandem, and similar oblique paths for 3 lateral oblique needles (LO) in direction of lateral parametrium on either side. There are two channels for lateral straight needles on each side. Additionally, there are similar tunnels for one anterior straight needle and two posterior straight needles (Figure 1). The intracavitary tandem straighten the uterus and helps to stabilize the whole application; it is also required for IC type source loading. This template with the central tandem and planned catheters allows the coverage of distal parametria (up to $4.5 \mathrm{~cm}$ from the middle of the IC tube) at the level of point A and the upper third of vagina.

A customized lock was devised that will prevent rotational and translational motion between the central tandem and the cylinder. With the availability of the MR compatible tandem, the applicator will be fully MR compatible.

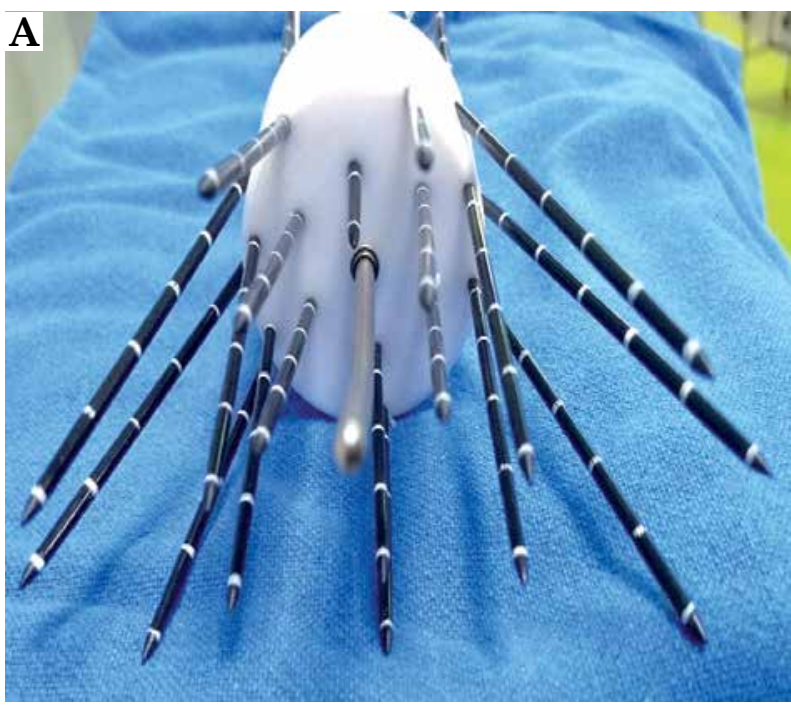

Fig. 1. A, B) The Medanta AOLO applicator

\section{Clinical indications}

This new template can be used for locally advanced cervical carcinomas with bulky lateral component, in patients having poor response to external beam radiotherapy, or disease that has invaded the bladder or rectum (stage IVA). In addition, this applicator can be used in patients who are candidates for simple standard intra cavitary radiotherapy (ICRT).

Here below we present a clinical situation where the template was successfully implemented after Institutional review board permission and ethics committee clearance was granted.

A 65-year-old lady with cervical cancer IIIB was taken for brachytherapy. Patient had grade 3 hematological toxicity during external beam radiotherapy, which delayed the start of brachytherapy for 7 days. Clinical examinations and magnetic resonance imaging (MRI) at diagnosis and after external beam radiotherapy completion were available to preplan HRCTV. A schematic clinical cartoon was drawn as per method described previously [6]. The maximum height, width, and thickness of HRCTV was found to be $6,6.7$, and $3.7 \mathrm{~cm}$, respectively. A preplanning was done and number of needles were determined to treat the lateral disease.

Under standard positioning, a Foley catheter inflated with $7 \mathrm{cc}$ of saline solution was placed in the bladder. The uterus was sounded and the central tandem was inserted. Then, the template was placed from the rear end of the tandem and pushed to the cervical os till it was comfortably positioned in the upper $3^{\text {rd }}$ of vagina. Ultrasonography (USG) probe was used in the operating room to identify HRCTV and proper placement of the central tandem and the needles, since USG is an established modality for real-time guidance in gynecologic brachytherapy [8]. The applicator was positioned in a proper orientation and then, the medial straight AO needles were inserted, after which, the lateral straight needles were implanted. The LO divergent needles were inserted subsequently. There are holes for anterior and posterior straight needles, but needs to be inserted depending on the severity of dis-

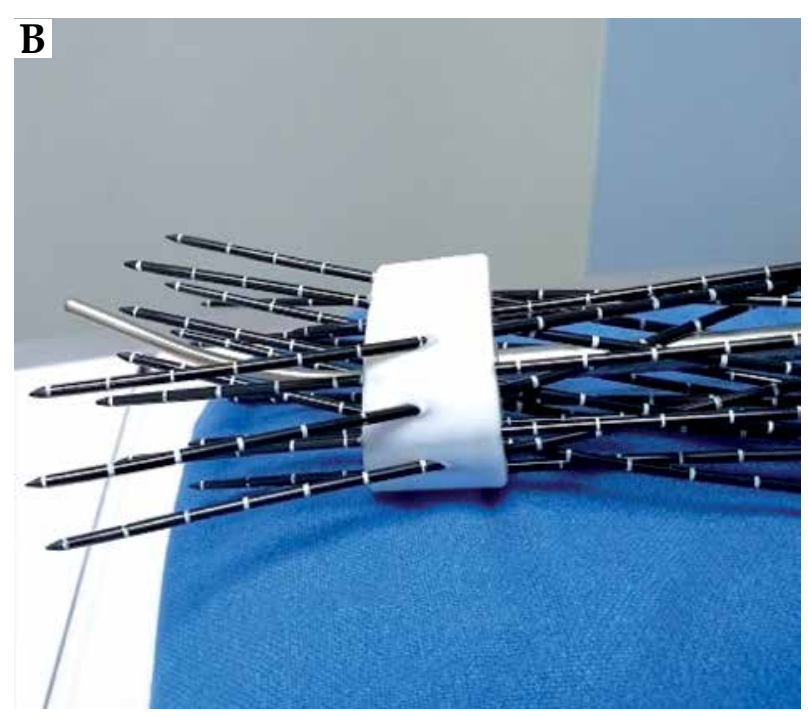


ease and the volume of HRCTV. The farthest posterior needle should be inserted with major caution and only if required, as it may perforate the rectum. Different diameter of vaginal cylinders $(3,4$, and $5 \mathrm{~cm})$ are available to match different anatomies.

Afterwards, a computed tomography (CT) planning scan was done. The Nucletron Oncentra MasterPlan brachytherapy planning system version 4.5 .3 was used for contouring and brachytherapy planning. Needles were reconstructed, and HRCTV was drawn from clinical examination and the pre-brachytherapy T2 MRI images.

\section{Planning}

First, a standard ICRT source loading pattern was followed, with one position in each AO needle replicating source positions in an ovoid (the Manchester system). Then, as advised by GEC-ESTRO group, the LS and LO needles were activated keeping no loading more than $10-20 \%$ from the ovoid source position by manual optimization [9]. The loading pattern gives coverage of $95 \%$ of the prescription dose for HRCTV.

The customary brachytherapy approach adopted by our department was 6 Gy $\times 4$ fractions, 2 fractions with each insertions in a gap of more than 6 hours. The aim of the treatment plan was to deliver at least 75-85 Gy $\mathrm{EQD}_{2}(\alpha / \beta=10)$ of dose to more than $90 \%$ of volume of HRCTV. Total doses when normalized to 2 Gy per fraction equivalent doses $\left(\mathrm{EQD}_{2}\right)$, assuming $\alpha / \beta$ of 10 for HRCTV the resultant EQD 2 for HRCTV was $75 \mathrm{~Gy}$. Dose constraints applied to the OARs were based on the EMBRACE II protocol for cervix. The details are presented in Table 1. Findings from two implants of the same patient are tabulated, showing volumes of HRCTV, dose coverage, and dose received by critical structures. The final EQD 2 and conformity index has also been calculated. Implant with the needles were removed under anesthesia after the treatment of second day in each implant. Regular antibiotics and intravenous analgesia (acetaminophen and tramadol in routine doses) were sufficient to control pain and to prevent infections. Anti-embolism stockings were prescribed during the treatment period. The patient was discharged on the same day of implant removal.

\section{Results}

The purpose of our manuscript is to describe the newly designed template and its efficacy in CTV coverage achieved with the IC/IS technique. Also, the Medanta template qualifies for treating locally advanced cancer cervix with lateral parametrial extent of disease (Figure 2).

The applicator is very easy to use and can give desired dose distributions with confidence; the very first application took only 30 minutes. In the future phase of this project, we will treat more cases with the applicator and the results will be reported.

\section{Discussion}

Combined intracavitary and interstitial BT with volumetric image-based treatment planning should be performed for patients with residual parametrial disease beyond point A. Dose escalation $>84 \mathrm{~Gy}$ is recommended in this patients' cohort. The ABS encourages interstitial techniques to be used in the developing world, where advanced cases are mostly found. The need of indige-

Table 1. Results of two clinical applications

\begin{tabular}{lccccccc} 
& $\begin{array}{c}\text { HRCTV volume } \\
\text { (cc) }\end{array}$ & Bladder 2 cc & Rectum 2 cc & Sigmoid 2 cc & HRCTV V $_{90}$ & COIN \\
\hline Application 1 & $55 c c$ & 11.0 Gy & 9.36 Gy & 8.6 Gy & $97 \%$ & 0.79 \\
\hline Application 2 & $54 c c$ & 11.4 Gy & 8.96 Gy & 8.5 Gy & $96 \%$ & 0.84 \\
\hline $\begin{array}{l}\mathrm{EQD}_{2} \text { (Gy) } \\
\text { (EBRT + brachytherapy) }\end{array}$ & 75 & 80.9 & 74.3 & 72.24 & 75 & NA
\end{tabular}

EBRT - external beam radiotherapy, CC - cubic centimeter, EQD -2 Gy equivalent dose: a/b for rectum, bladder, and sigmoid is 3, for HRCTV is 10. COIN - conformal index $=P T V P D / V P T V \times P T V P D / V P D, P T V P D-P T V$ receiving prescription dose, VPD - target volume receiving prescription dose, VPTV - volume of PTV
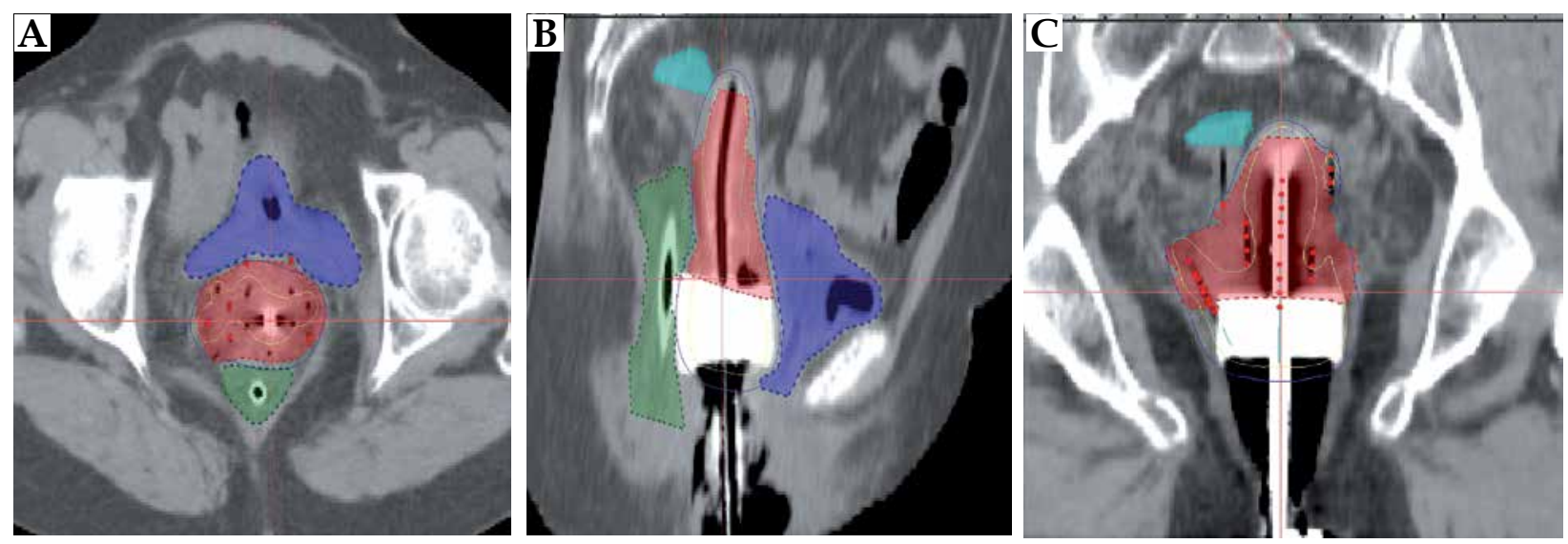

Fig. 2. The Medanta AOLO applicator in clinical use 
nous development of applicator and its clinical use has been mentioned previously [10]. A recent survey in India shows that while more than $50 \%$ centers use CT as volumetric imaging, $87 \%$ centers do not perform any interstitial procedures [11]. The lack of affordable interstitial applicators is a significant reason. The picture is quite similar in developing world [4]. The ABS has recommended $\mathrm{CT}$ and/or MRI as primary imaging for IGABT in advanced cervical cancer patients. Furthermore, the Indian Brachytherapy Society guidelines published recently also states that CT can be used as an alternative imaging [12].

The widespread use of such low cost templates and CT scans will help more centers to access the benefits of IGABT for their patients. IGABT leads to a significant improvement in local control rates and disease free survival in LACC, and should be considered for wider implementation in developing countries to improve outcomes. It is worth to mention that our $3 \mathrm{~cm}$ diameter of intra-vaginal template is possibly the first of its kind, and could be helpful for patients having narrow vagina. The only feature we found to be carefully addressed is the tagging of the needles. As compared to standard applicators, the number of needles are few more and may be difficult to number if not routinely done at the time of insertion. However, numbering of needles is a standard procedure in all centers using this technique. As of now, we believe that, with further clinical experiences we will be able to deliver higher dose to HRCTV with better understanding of the strengths and weakness of the applicator in the near future.

\section{Conclusions}

We have created a template that is presently CT compatible and in the near future, it will be MR compatible with incorporation of the tandem. The template/applicator can be used to treat advanced cervical cancers by IGABT as per GEC ESTRO guidelines.

\section{Acknowledgments}

The authors gratefully remembers the teachings of AROI-ESTRO teachers during the AROI ESTRO courses held in India.

\section{Disclosure}

The authors report no conflict of interest.

\section{References}

1. Pötter R, Haie-Meder C, Van Limbergen E et al. Recommendations from Gynaecological (GYN) GEC ESTRO Working Group (II): concepts and terms in 3D image based treatment planning in cervix cancer brachytherapy - 3D dose volume parameters and aspects of 3D image-based anatomy, radiations physics, radiobiology. Radiother Oncol 2006; 78: 67-77.

2. Tan LT, Tanderup K, Kirisits $C$ et al. Image-guided adaptive radiotherapy in cervical cancer. Semin Radiat Oncol 2019; 29: 284-298.

3. Deshpande DD. Will MR image-guided brachytherapy be a standard of care for cervical cancer in future? An Indian perspective. J Med Phys 2012; 37: 1-3.
4. Suneja G, Brown D, Chang A et al. American Brachytherapy Society: Brachytherapy treatment recommendations for locally advanced cervix cancer for low-income and middle-income countries. Brachytherapy 2017; 16: 85-94.

5. Banerjee S, Mahantshetty U, Shrivastava S. Brachytherapy in India - a long road ahead. J Contemp Brachytherapy 2014; 6: 331-335.

6. Banerjee S, Richard P, Christian K et al. 3D mapping for precise definition of GTV, CTV and their correlation in cervix cancer BT (EMBRACE). Radiat Oncol 2017; 123: S520-521 [Abstract].

7. Venkatesan K, Banarjee S, Kataria T et al. Dosimetric analysis of indigenously made intracavitary and interstitial (IC + IS) gynecological applicator in image-based brachytherapy. Med Phys Int J 2019; 7: 372 [Abstract].

8. Banerjee S, Kataria T, Gupta D et al. Use of ultrasound in image-guided high-dose-rate brachytherapy: enumerations and arguments. J Contemp Brachytherapy 2017; 9: 146-150.

9. Viswanathan AN, Beriwal S, De Los Santos et al. American Brachytherapy Society consensus guidelines for locally advanced carcinoma of the cervix. Part II: high-dose-rate brachytherapy. Brachytherapy 2012; 11: 47-52.

10. Villalba SR, Sancho JR, Palacín AO et al. Development and clinical implementation of a new template for MRI-based intracavitary/interstitial gynecologic brachytherapy for locally advanced cervical cancer: from CT-based MUPIT to the MRI compatible Template Benidorm. Ten years of experience. J Contemp Brachytherapy 2016; 5: 404-414.

11. Bandyopadhyay A, Basu P, Roy K et al. Treatment of locally advanced carcinoma cervix with special emphasis on brachytherapy: A practice pattern survey among young radiation oncologist of India. South Asian J Cancer 2018; 7: 231235.

12. Mahantshetty U, Gudi S, Singh R et al. Indian Brachytherapy Society Guidelines for radiotherapeutic management of cervical cancer with special emphasis on high-dose-rate brachytherapy. J Contemp Brachytherapy 2019; 11: 293-306. 